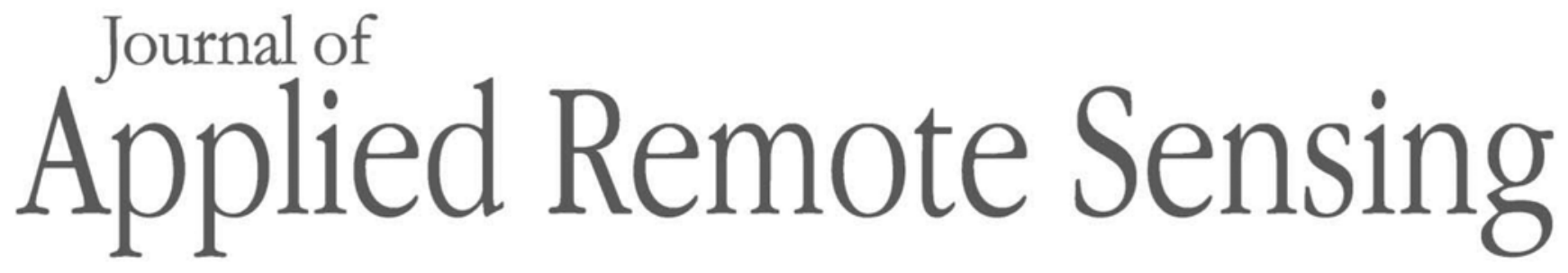

RemoteSensing.SPIEDigitalLibrary.org

\title{
Modified MODIS fluorescence line height data product to improve image interpretation for red tide monitoring in the eastern Gulf of Mexico
}

Chuanmin $\mathrm{Hu}$ Lian Feng 


\title{
Modified MODIS fluorescence line height data product to improve image interpretation for red tide monitoring in the eastern Gulf of Mexico
}

\author{
Chuanmin Hu* and Lian Feng \\ University of South Florida, 140 Seventh Avenue South, St. Petersburg, Florida 33701, \\ United States
}

\begin{abstract}
Several satellite-based methods have been used to detect and trace Karenia brevis red tide blooms in the eastern Gulf of Mexico. Some require data statistics and multiple data products while others use a single data product. Of these, the MODIS normalized fluorescence line height (nFLH) has shown its advantage of detecting blooms in waters rich in colored dissolved organic matter, thus having been used routinely to assess bloom conditions by the Florida Fish and Wildlife Conservation Commission (FWC), the official state agency of Florida responsible for red tide monitoring and mitigation. However, elevated sediment concentrations in the water column due to wind storms can also result in high nFLH values, leading to false-positive bloom interpretation. Here, a modified nFLH data product is developed to minimize such impacts through empirical adjustments of the nFLH values using MODIS-derived remote sensing reflectance in the green band at $547 \mathrm{~nm}$. The new product is termed as an algal bloom index (ABI), which has shown improved performance over the original $\mathrm{nFLH}$ in both retrospective evaluation statistics and near real-time applications. The ABI product has been made available in near realtime through a Web portal and has been used by the FWC on a routine basis to guide field sampling efforts and prepare for red tide bulletins distributed to many user groups. (c) The Authors. Published by SPIE under a Creative Commons Attribution 3.0 Unported License. Distribution or reproduction of this work in whole or in part requires full attribution of the original publication, including its DOI. [DOI: 10.1117/1.JRS.11.012003]
\end{abstract}

Keywords: Karenia brevis; harmful algal blooms; monitoring; moderate resolution imaging spectroradiometer; fluorescence line height; colored dissolved organic matter; West Florida Shelf; Gulf of Mexico.

Paper 16576SS received Aug. 3, 2016; accepted for publication Nov. 28, 2016; published online Dec. 20, 2016.

\section{Introduction}

Harmful algal blooms (HABs) of the toxic dinoflagellate, Karenia brevis, occur annually on the West Florida shelf (WFS) in the eastern Gulf of Mexico (GOM) ${ }^{1}$ often causing fish and marine mammal mortality as well as other environmental and economic problems. ${ }^{2}$ Timely information of $K$. brevis blooms in terms of their spatial extent, intensity, transport, and dissipation provides guidance on field sampling and mitigation efforts, and retrospective information on such bloom characteristics helps understand bloom formation and their impact on the ocean's biophysical and biogeochemical properties. Satellite remote sensing has proven to be an effective tool to provide such information because $K$. brevis blooms cause water discoloration, making bloom waters greenish, brownish, or reddish as compared with the nearby nonbloom waters. For the reason of discoloration, K. brevis blooms are often called "red tides." The discoloration is caused primarily by the light absorption pigments in the dinoflagellate cells. Although the pigment concentration varies among cells, typically a concentration of 50,000 cells $\mathrm{L}^{-1}$ is equivalent to about $0.5 \mu \mathrm{g} \mathrm{L}^{-1}$ chlorophyll, enough to be differentiated from the typical background concentrations

*Address all correspondence to: Chuanmin Hu, E-mail: huc@usf.edu 
of 0.1 to $0.2 \mu \mathrm{g} \mathrm{L}-1$ in most of surface waters on the WFS. In some offshore waters during certain seasons, the background concentration may be $0.1 \mu \mathrm{g} \mathrm{L}-1$ or even lower, making it possible to detect $K$. brevis concentrations of $>20,000$ cells L ${ }^{-1}$ (corresponding to about $0.2 \mu \mathrm{g} \mathrm{L}^{-1}$ ) in such clear waters. In the GOM, background concentrations of $K$. brevis are below 1000 cells L $^{-1}$. Above 5000 cells $\mathrm{L}^{-1}$, commercial shellfish areas are mandated to close for harvesting according to regulations of the U.S. Food and Drug Administration. ${ }^{3}$ At $\sim 10^{5}$ cells $\mathrm{L}^{-1}$, fish kills are often

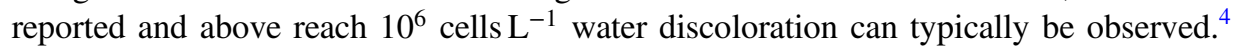

Because of the chlorophyll content in the $K$. brevis cells, most remote sensing techniques have relied on the use of satellite ocean color sensors because these sensors were designed to estimate chlorophyll concentrations in surface waters. The most often used sensors for the GOM include the sea-viewing wide field-of-view sensor (SeaWiFS, 1997 to 2010), medium resolution imaging spectrometer (2002 to 2012), moderate resolution imaging spectroradiometer (MODIS, 2000 to present on Terra and 2002 to present on Aqua), and the most recent visible-infrared imager radiometer suite (VIIRS, 2011 to present). Although their sensor characteristics differ from each other (e.g., signal-to-noise ratio, number of spectral bands, band widths, band centers, spatial resolution, swath width, and revisit frequency), the principle is the same: to detect and quantify the color changes in certain spectral bands, which are further related to changes in Chl or $K$. brevis cell concentrations. Here, the term "color" refers to the spectral remote sensing reflectance $\left(\mathrm{Rrs}, \mathrm{sr}^{-1}\right)$ or normalized water-leaving radiance $\left(\mathrm{nLw}, \mathrm{mW} \mathrm{cm}^{-2} \mu \mathrm{m}^{-1} \mathrm{sr}^{-1}\right)$ after atmospheric correction of the calibrated sensor signal.

Although the principle is the same, several different methods have been developed for bloom detection and tracking. ${ }^{5-10}$ In these methods, a bloom is first detected and then determined whether it is mainly dominated by $K$. brevis. The former requires the use of algorithms with either blue/green bands ${ }^{9}$ or near-infrared/red bands, ${ }^{5,8}$ whereas the latter is done through inspection of the backscattering efficiency, ${ }^{6}$ spectral curvature, ${ }^{10}$ or $\mathrm{nLw}$ in the green. ${ }^{7}$ Recently, a thorough review has been conducted to evaluate all of these different methods ${ }^{11,12}$ and concluded that they all perform similarly with different strengths and weaknesses for different conditions.

Among these various methods, two have been used routinely by the U.S. federal and state agencies for bloom monitoring in the GOM. The first has been used by the U.S. National Oceanic and Atmospheric Administration (NOAA) through its HAB bulletin, ${ }^{13}$ which is based on the Chla-anomaly method ${ }^{9}$ to detect "new" bloom patches and on the spectral curvature method to discriminate K. brevis blooms. ${ }^{10}$ The second has been used by the Florida Fish and Wildlife Conservation Commission (FWC), the official agency of the state of Florida responsible for red tide monitoring and mitigation. It is based on the MODIS normalized fluorescence line height $\left(\mathrm{nFLH}=\mathrm{FLH}\right.$ normalized to solar irradiance) imagery to detect bloom patches, ${ }^{8}$ and then on field measurements to discriminate K. brevis blooms. ${ }^{14}$ These MODIS and field-based detections are first interpreted by human analysts and summarized in HAB bulletins, and then distributed to various stakeholders through e-mail subscriptions after combining results from numerical models. The combination of these products has essentially formed an integrated red tide monitoring system to provide relevant information in near real-time through a web portal. $^{15}$

However, one shortcoming in the use of MODIS nFLH to detect blooms is its sensitivity to inorganic particles (i.e., resuspended sediments). Through observations and model simulations, it is well known that nFLH is insensitive to perturbations by colored dissolved organic matter (CDOM, mostly terrestrial origin on the WFS) ${ }^{8,16-18}$ A 20-fold increase in CDOM will only decrease $\mathrm{nFLH}$ by half. ${ }^{18}$ The same observations and simulations also suggest strong sensitivity of nFLH to resuspended sediments, causing false-positive bloom detection. On the WFS, wind storms from hurricanes or cold fronts can cause significant sediment resuspension. In the past, this problem has been circumvented by examining another type of MODIS imagery, namely enhanced red-green-blue (ERGB) imagery. ${ }^{8,19}$ This is because sediment plumes in ERGB imagery appear bright while bloom waters appear dark, making it straightforward to differentiate the two. However, this involves inspection and presentation of two image types, often making it difficult for most users, especially when they are untrained for image interpretation. Although some alternative ways have been proposed to overcome this difficulty through band subtraction or band normalization, ${ }^{5,7}$ they have not been implemented for near real-time production due to extra steps required in satellite data processing and quality control. While in the long run they 
may eventually be implemented and tested for operational use, for continuity of the FWC bulletins the perturbation of suspended sediments to MODIS nFLH needs to be addressed.

It is thus desirable to develop a new product to take the full advantage of the MODIS nFLH (i.e., readily available from standard data processing, tolerant to $\mathrm{CDOM}$ perturbation, routine use in FWC HAB bulletins) while minimizing the impact of resuspended sediments. The objective of this paper is, therefore, not to compare the performance of different bloom detection methods as such as a comparison has already been performed elsewhere, ${ }^{9,10}$ but to develop and validate such a new product to serve as an algal bloom index (ABI), with the ultimate goal to replace the default MODIS nFLH to improve image interpretation and bloom detection for near realtime applications.

\section{Data and Method}

\subsection{Satellite Data}

MODIS level-0 data were obtained from NASA Goddard Space Flight Center (GSFC). ${ }^{20}$ The purpose of starting from level-0 instead of level-2 was to generate several nonstandard data products used in the integrated red tide information system (IRIS), ${ }^{15,21}$ e.g., imagery generated from Rayleigh-corrected reflectance (Rrc, dimensionless). Level-0 data were processed using the NASA standard processing package (SeaDAS) to generate both standard and nonstandard level-2 products. The former include nFLH (i.e., FLH normalized to solar irradiance inputs), spectral Rrs, spectral $\mathrm{nLw}$, and data quality control flags. The latter includes spectral Rrc and products (e.g., red-green-blue imagery to visualize and floating algae inde ${ }^{22}$ to detect floating macroalgae and Trichodesmium) generated from Rrc. All data products were mapped to a cylindrical-equidistant projection at $\sim 1-\mathrm{km}$ resolution through resampling.

The NASA standard product of $\mathrm{nFLH}$ is derived as

$$
\mathrm{nFLH}=\mathrm{nLw}(678)-\{\mathrm{nLw}(667)+[\mathrm{nLw}(754)-\mathrm{nLw}(667)] \times(678-667) /(754-667)\} .
$$

Based on nFLH and spectral Rrs, three other indices have been developed for red tide monitoring. The first two are red blue difference (RBD) and Karenia brevis bloom index (KBBI) from Amin et al. ${ }^{5}$

$$
\begin{gathered}
\mathrm{RBD}=\mathrm{nLw}(678)-\mathrm{nLw}(667), \\
\mathrm{KBBI}=[\mathrm{nLw}(678)-\mathrm{nLw}(667)] /[\mathrm{nLw}(678)+\mathrm{nLw}(667)] .
\end{gathered}
$$

In Ref. 5, a pixel meeting the following criteria was classified as a $K$. brevis bloom pixel: $\mathrm{RBD}>0.015 \mathrm{~mW} \mathrm{~cm}{ }^{-2} \mu \mathrm{m}^{-1} \mathrm{sr}^{-1}$, and KBBI $>3 \times \mathrm{RBD}$.

The last index, to be developed in this study [Eq. (4)], is an ABI. It is basically the same nFLH index but modulated by Rrs(547) to minimize the influence of suspended sediments. Similar empirical approaches have been developed by Wynne et al. ${ }^{23}$ to empirically adjust satellite-derived chlorophyll concentration $\left(\mathrm{Chl}, \mu \mathrm{g} \mathrm{L}^{-1}\right)$ in sediment-rich waters and by Qi et al. ${ }^{24}$ to empirically adjust satellite-derived maximum chlorophyll index in sediment-rich waters. The idea is to add an extra term to adjust nFLH for sediment-rich waters, where the term has minimal influence on $\mathrm{nFLH}$ for sediment-poor waters. In this work, the following considerations were used to design the empirical fix.

While the relative abundance of sediment can be expressed through Rrs in the red band, e.g., Refs. 25-28, in practice Rrs in the red is noisy for clear waters. Therefore, to retain image quality for both sediment-rich and sediment-poor waters, the red band was not used. Instead, Rrs in the green (547 nm for MODIS) was used for the adjustment of nFLH. This is because Rrs(547) is relatively a constant for clear waters with $\mathrm{Chl}<0.25 \mu \mathrm{g} \mathrm{L}^{-1}$ (Ref. 29) but it is also sensitive to changes in sediment concentrations in coastal waters. Actually, Carvalho et al. ${ }^{7}$ suggested the use of water-leaving radiance ( $\mathrm{Lw})$ in the green band to "normalize" Chl, yet $\mathrm{Lw}$ is a function of 
solar/viewing geometry and atmospheric transmittance, thus could change with time. The use of $\operatorname{Rrs}(547)$ would avoid this problem.

By convention, a normalization is usually through division against the reference, thus a normalized nFLH against Rrs(547) would have the form of $n F L H / R r s(547)$. In practice, however, this would lead to very high $\mathrm{nFLH/Rrs(547)}$ values for clear waters because Rrs(547) for clear water is very low and nearly a constant of $\sim 0.0015 \mathrm{sr}^{-1} .{ }^{29}$ Therefore, to avoid this problem, the normalization was designed to take the following form:

$$
\mathrm{ABI}=\mathrm{nFLH} /\{1+[\operatorname{Rrs}(547)-0.0015] \times \alpha\} .
$$

Here, nFLH and Rrs(547) are the default MODIS data products from SeaDAS processing, $0.0015\left(\mathrm{sr}^{-1}\right)$ is a constant representing $\operatorname{Rrs}(547)$ for clear waters, and the empirical factor $\alpha$ (units: sr) is used to normalize nFLH against $\operatorname{Rrs}(547)$. The empirical factor was adjusted through trial-and-error until a best match is found between ABI and field-measured cell counts data (see below). In this study, $\alpha$ was determined to be 80 . For other regions that also suffer from similar problems of "contamination" by suspended sediments, $\alpha$ may be different but can be determined the same way once field data are available.

Note that by taking this empirical adjustment, ABI is basically the same as nFLH for clear waters where $\operatorname{Rrs}(547) \sim 0.0015 \mathrm{sr}^{-1}$, and ABI also has the same units as nFLH $\left(\mathrm{mW} \mathrm{cm} \mathrm{cm}^{-2} \mu \mathrm{m}^{-1} \mathrm{sr}^{-1}\right.$. For turbid waters where $\operatorname{Rrs}(547)$ is $\gg 0.0015 \mathrm{sr}^{-1}$, ABI is $\ll \mathrm{nFLH}$, thus greatly reducing the false-positive signals caused by sediment resuspension. The validity of $\mathrm{ABI}$ and its improvement over the original $\mathrm{nFLH}$ are shown below through point validations and image inspections.

\subsection{Field Data}

As the official state agency responsible for red tide monitoring and mitigation, the FWC has maintained a state-wide ret tide monitoring program, where periodic sampling of coastal waters from either dedicated research groups or volunteers has been conducted. In these sampling efforts, surface water samples were collected with a 2.2-L beta sampling bottle (Wildco, Yulee, Florida). From each bottle, $250 \mathrm{~mL}$ subsamples were preserved in Lugol's iodine, ${ }^{30}$ and then sent to the FWC lab for phytoplankton enumeration to determine cell concentrations. During the enumeration, the subsamples were examined with microscopy within $24 \mathrm{~h}$ of sample collection, where identification and enumeration of HAB species were performed under $200 \times$ magnification using either a Olympus DP70 inverted microscope or a Zeiss Axiovert 100. ${ }^{31}$ For cell enumeration, $3 \mathrm{~mL}$ of each preserved sample was settled for $>30 \mathrm{~min}$ in a Lab-Tek coverslip bottom chamber (Nalgene-Nunc \#155379) ${ }^{32}$ HAB species in the entire chamber were identified to the lowest taxonomic level possible and enumerated. Figure 1 shows the locations of some of the water samples collected between 2002 and 2012, where same-day MODIS data were also collected over the same locations.

\subsection{Validation}

Two methods were used to evaluate the newly developed MODIS ABI: pixel-wise comparison with concurrent cell counts data and inspection of image pairs between nFLH and ABI from typical bloom and nonbloom cases on the WFS.

For the first method, matching pairs between MODIS satellite observations and field samples have been obtained using two criteria: (1) MODIS/Aqua data were acquired on the same day of the in situ measurements and (2) variance of $3 \times 3$ MODIS pixels centered at the in situ station was $<10 \%$ to assure that water was relatively homogeneous. A total number of 557 matching pairs were found after applying the two criteria, with locations shown in Fig. 1. As the background $\mathrm{Chl}$ of the offshore GOM waters is $\sim 0.1 \mu \mathrm{g} \mathrm{L}-1$, corresponding to $\sim 10^{4}$ cells L ${ }^{-1}$ (Ref. 8) (this is actually the threshold K. brevis concentration above which the class of "low" bloom condition is defined by the FWC) only the 145 matching pairs with $K$. brevis cell concentrations of $>10^{4}$ cells $\mathrm{L}^{-1}$ were used for validation in this study. Note that the actual detection threshold may be much higher than $10^{4}$ cells $\mathrm{L}^{-1}$ unless the bloom is a mixture of $K$. brevis and 


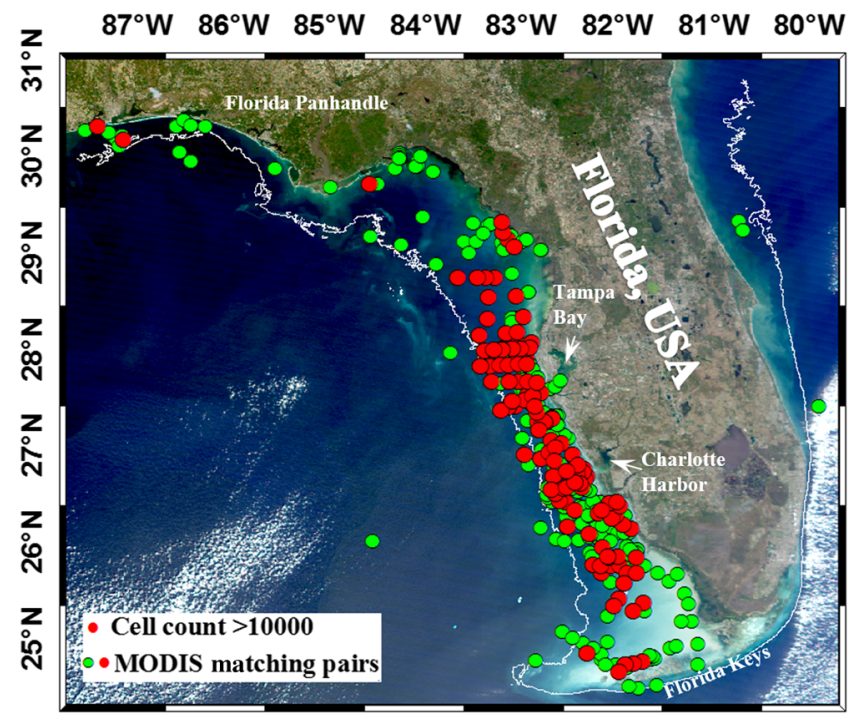

Fig. 1 Locations of the matching pairs between MODIS/Aqua observations and FWC field samples, where stations with $K$. brevis cell concentrations of $>10^{4}$ cells $\mathrm{L}^{-1}$ are annotated in red. The data were collected between 2002 and 2012. The use of $10^{4}$ as the threshold was because the FWC has been using $10^{4}$ to $10^{5}$ cells $\mathrm{L}^{-1}$ to represent "low" $\mathrm{K}$. brevis concentrations that are one order of magnitude higher than the "presence" concentration. The background is a MODIS/Terra RGB true color composite image on February 13, 2011. The white line indicates the 30-m bathymetric isobath. Several places along the west coast of Florida are annotated to facilitate image interpretation in the following images.

other phytoplankton such as diatoms, but to follow the FWC convention to define "low concentration bloom" the threshold of $10^{4}$ cells $\mathrm{L}^{-1}$ was used here. For comparison, a threshold of $2 \times 10^{4}$ cells $\mathrm{L}^{-1}$ was also used. In the comprehensive analysis of Soto et al., ${ }^{12}$ the bloom threshold was based on the $\mathrm{nFLH}$ value of $0.033 \mathrm{~mW} \mathrm{~cm}{ }^{-2} \mu \mathrm{m}^{-1} \mathrm{sr}^{-1}$. Here for comparison, correlation analysis was used to examine the relationships between $K$. brevis cell concentrations of water samples and the corresponding MODIS-derived nFLH, ABI, RBD, and KBBI using these matching pairs, and regression was used to estimate cell concentrations from ABI.

For the second method, typical cases on the WFS that could potentially be falsely recognized as red tide blooms include hurricane- or other wind-induced sediment resuspension, and river plumes. Therefore, each of these cases was selected to examine the effectiveness of ABI in ruling out these false positives. For reference, a case of $K$. brevis bloom without the perturbations of sediment resuspension and river plumes was also selected. The cases were selected when concurrent field data were available to confirm the visual interpretations. For each case, the following images were selected for visual inspection: (1) MODIS nFLH; (2) MODIS ERGB (to differentiate sediment-rich from sediment-poor waters, and to differentiate optically shallow from optically deep waters); (3) MODIS RBD; and (4) MODIS ABI. The selection of visual inspection as the method for validation is because (1) MODIS imagery has been interpreted visually together with concurrent field data by the FWC to determine the spatial extend of red tide, and such information was summarized in HAB bulletins and distributed to various user groups through e-mail subscription. ${ }^{15}$ This is also how NOAA determined red tide spatial extent in the NOAA HAB bulletins; (2) red tide is very patchy, ${ }^{10}$ making a pixel-wise validation subject to large uncertainties. In practice, once one or more water samples from the satelliteidentified bloom patch confirmed high concentration of $K$. brevis, the entire bloom patch was inferred to be $K$. brevis bloom. This type of visual validation has been a standard method used by many agencies to verify whether a patch of color anomaly in satellite imagery is $K$. brevis bloom. Note that because the FWC releases field data and HAB bulletins once a week, the time window to show "concurrent" field data and MODIS imagery was selected to be \pm 7 days. Given the typical retention periods of $K$. brevis HABs of $>1$ month (can reach 6 months), the 7-day time window is unlikely to cause major mismatches between field and MODIS observations. 


\section{Results}

\subsection{Pixel-Wise Validation}

Figure 1 shows where concurrent MODIS and field data were collected between 2002 and 2012 (red dots). Most of the field data were from shallow waters $(<30 \mathrm{~m})$ on the WFS where most red tides have been reported, but some data were from the Florida Keys and the Florida Panhandle. The synoptic coverage of the field data suggests that the data used in this study can represent most, if not all, oceanographic conditions such as blooms, river plumes, sediment resuspension, and optically shallow waters.

Figure 2 shows the comparison between $K$. brevis cell concentrations and four MODIS indices (nFLH, ABI, RBD, and HBBI) over the same locations on the same day. Overall, despite the large data scattering due to $K$. brevis bloom patchiness and the difficulty in having an exact match between MODIS pixels $(1 \mathrm{~km})$ and field samples (a point) ${ }^{10}$ all four indices are significantly correlated with $K$. brevis cell concentrations. The correlation coefficient is the highest for ABI (0.57), followed by RBD (0.53), nFLH (0.52), and KBBI (0.49). In terms of bloom detection, they are about the same effective, with $\mathrm{ABI}$ being slightly better. What is more important is that some of the high-nFLH low-K. brevis points appear to be shifted to the left on the ABI-K. brevis plot, confirming that the design of Eq. (4) to avoid false positive in sediment-rich waters may be effective (i.e., high nFLH values are reduced in ABI). This observation is further verified in the case studies below.

\subsection{Case Studies}

Figures 3-7 show the five case study results, among which are, respectively:

1. Day 2003292: River Plume (see Fig. 3);

2. Day 2005300: Hurricane Wilma (see Fig. 4);

3. Day 2005361: Windstorm [maximum wind speed of $8.7 \mathrm{~m} / \mathrm{s}$ during this day, according to data collected from a National Data Buoy Center (NDBC) station in Long Key of Florida Keys, $24.844^{\circ} \mathrm{N}, 80.864^{\circ} \mathrm{W}$ ] (see Fig. 5);

4. Day 2003279: Mixed K. brevis bloom and diatom bloom (see Fig. 6);

5. Day 2006280: K. brevis bloom (see Fig. 7).

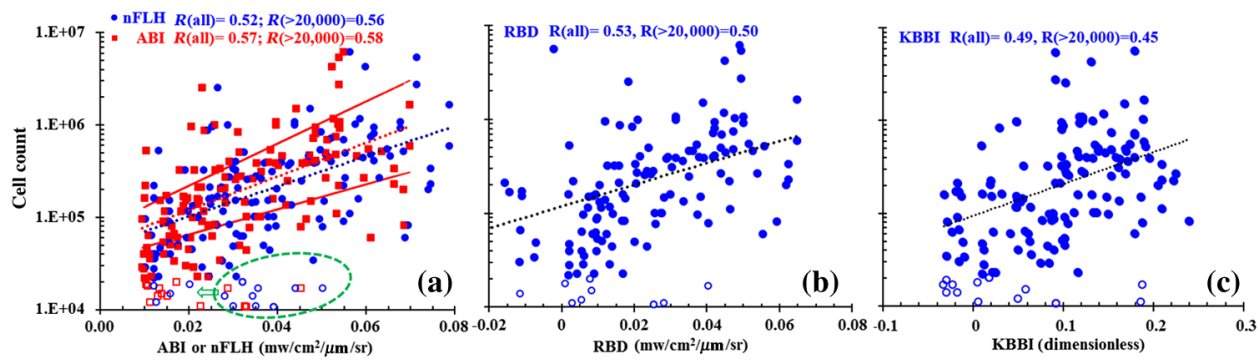

Fig. 2 Correlation between satellite derived nFLH: (a) $A B I$, (b) RBD, and (c) $K B B I$ and fieldmeasured $K$. brevis cell concentration for concentrations $>10^{4}$ cells $L^{-1}$ (all points, $N=145$ ) and for concentrations $>2 \times 10^{4}$ cells L ${ }^{-1}$ (solid points, $N=130$ ), respectively. The threshold of $10^{4}$ cells $\mathrm{L}^{-1}$ was to follow convention of FWC to classify "low counts" bloom, whereas the threshold of $2 \times 10^{4}$ cells L-1 (corresponding to about $0.2 \mu \mathrm{gL}^{-1}$ Chl for pure population) was for detection of blooms in clear waters where background Chl is $\sim 0.1 \mu \mathrm{g} \mathrm{L}^{-1}$. In Ref. 12, the $\mathrm{nFLH}$ threshold was set to $0.033 \mathrm{~mW} \mathrm{~cm}^{-2} \mu \mathrm{m}^{-1} \mathrm{sr}^{-1}$. Note that Amin et al. ${ }^{5}$ proposed to use $\mathrm{RBD}$ to detect blooms and then to use KBBI to classify the blooms (K. brevis or non-K. brevis), where threshold values were defined for both. Here for comparison, they are both evaluated against cell concentrations. The large data spread around the fitting lines is mainly due to bloom patchiness and the difficulty in having an exact match between MODIS pixels ( $1 \mathrm{~km})$ and field samples (a point). ${ }^{10}$ The dotted red line defines the regression (95\% confidence level marked with solid red lines) between $\mathrm{ABI}$ and cell counts, which is cell counts $=e^{41.918 * \mathrm{ABI}+10.856}$. Some points in $\mathrm{nFLH}$ (outlined in green) are shifted significantly to the left after normalizing to $\mathrm{ABI}$, indicating reduction in false-positive bloom detection. 


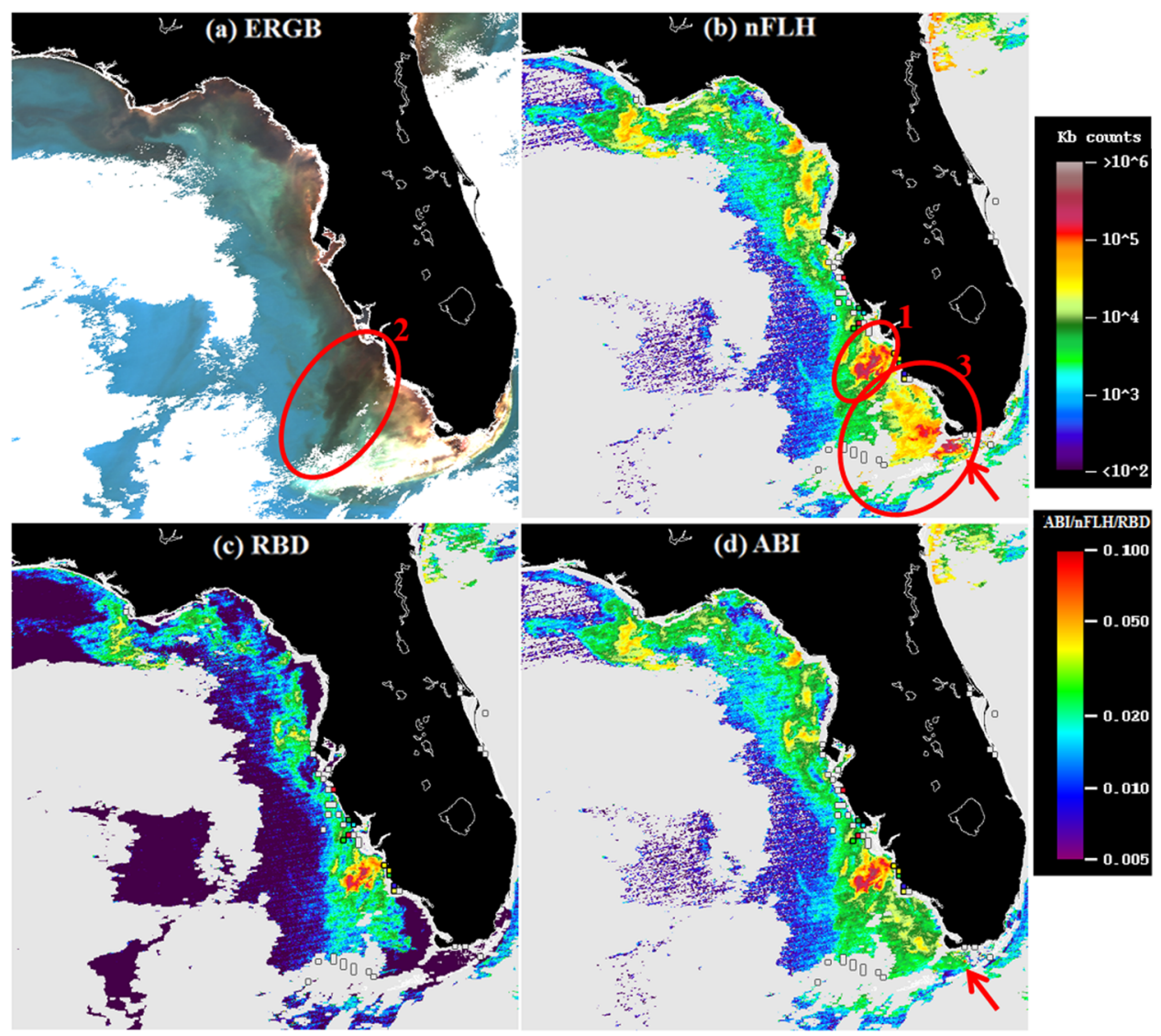

Fig. 3 The performance of three bloom indices in detecting a $K$. brevis bloom (outlined in circle 1) in a dark-water plume (outlined in circle 2), where MODIS images were acquired on day 292 of 2003. The plume has been reported in detail by Hu et al. ${ }^{33}$ as a result of excessive discharge from local rivers. The MODIS ERGB image in (a) shows the dark-colored plume, outlined in circle 2. The water appears dark in the ERGB image because of high absorption of blue light due to high concentrations of CDOM and/or phytoplankton in the plume. The MODIS nFLH image in (b) shows the bloom pattern (circle 1) within the dark plume, but it also shows high signal in sediment-rich waters (circle 3), causing false-positive detection. The RBD and ABI images in (c) and (d), respectively, successfully removed such a false-positive detection, but $A B I$ leads to more spatially coherent patterns than RBD (i.e., in sediment-rich waters RBD values are reversed to those of offshore clear waters). The arrows in (b) and (d) point to shallow-water $(<5 \mathrm{~m})$ locations in nearshore Florida Keys where the falsely elevated nFLH signals are reduced in ABI. Annotated in (b)(d) are K. brevis cell concentrations from water samples collected within 1 week ( \pm 7 days) of the MODIS image, with the concentration color-coded (top color legend). The bottom color legend applies to (b)-(d). Gray color for cell concentrations represents 0 counts $\mathrm{L}^{-1}$, and gray color on the MODIS image represents clouds, stray light, or saturation of the MODIS fluorescence bands.

While the case-specific interpretations can be found in the figure captions, a common finding from these case studies is that the high nFLH values in sediment-rich waters have always been dampened in the RBD and ABI images, thus reducing false-positive detection. Although a combination of ERGB and nFLH can serve for the same purpose, the FWC only uses one type of imagery to combine with field measurements to have interpreted results in the HAB bulletins to distribute to registered users. Therefore, the use of ABI as a replacement of nFLH is an effective way to improve image quality and bloom detection accuracy. What is important is that while the false-positive detection in sediment-rich waters is reduced, the ABI image patterns are nearly the same as the nFLH image patterns in sediment-poor waters, maintaining the effectiveness of the original $\mathrm{nFLH}$ in bloom detection. This is exactly the purpose of the ABI design in Eq. (4).

The use of RBD appears to be also effective in reducing false-positive detection, yet the spatial patterns in RBD imagery are less coherent than in ABI imagery, as RBD values from offshore to nearshore waters are reversed. Although this reversal does not impact 


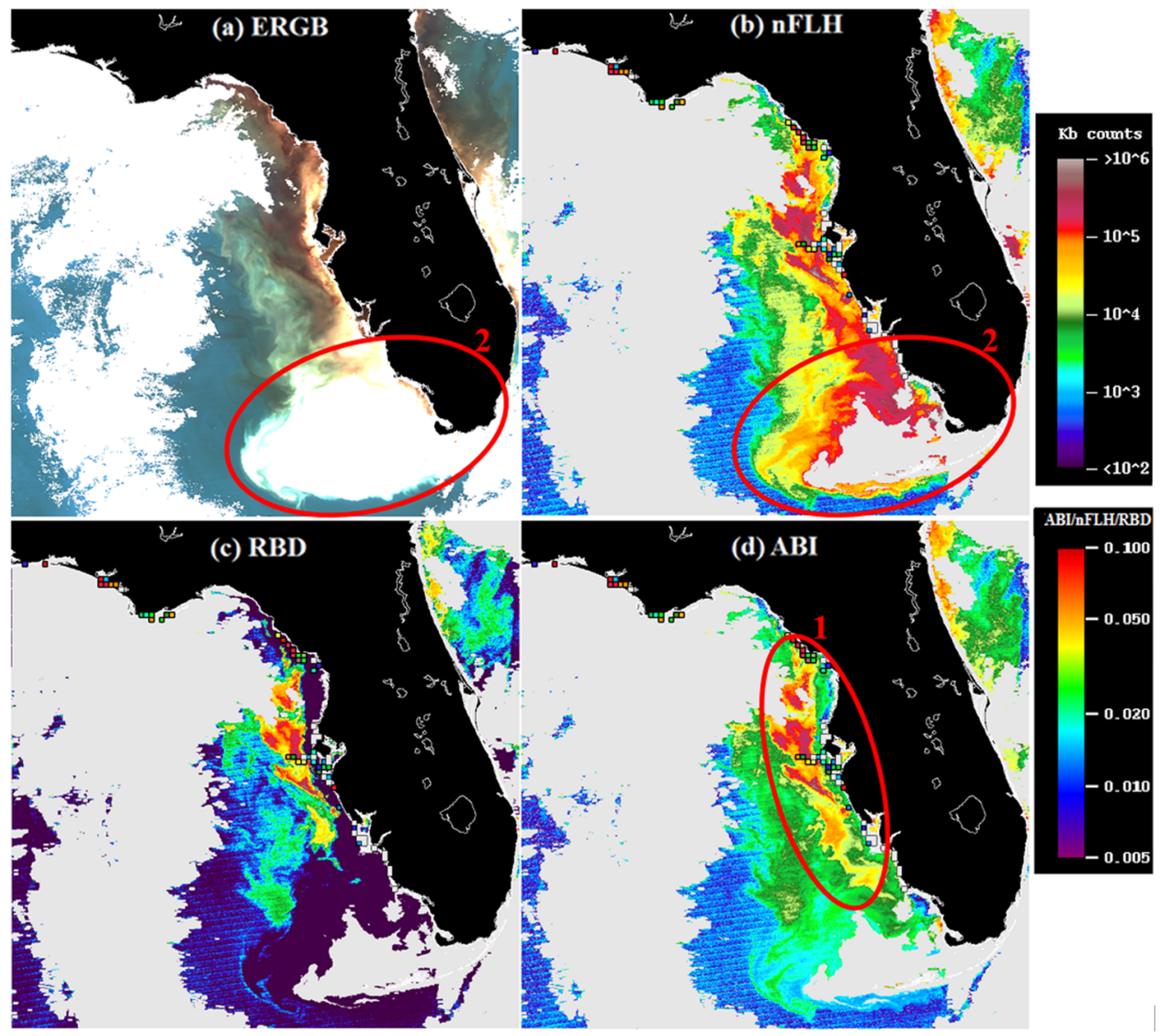

Fig. 4 The performance of three bloom indices in detecting a $K$. brevis bloom (outlined in circle 1) after the passage of Hurricane Wilma. The MODIS images were acquired on day 300 of 2005 . The event was reported in Ref. 34 (a) ERGB; (b) nFLH; (c) RBD; (d) ABI. Annotated in (b)-(d) are K. brevis cell concentrations from water samples collected within 1 week ( \pm 7 days) of the MODIS image, with the concentration color coded (top color legend). The bottom color legend applies to (b)-(d). Note that the false-positive detection in sediment-rich waters [outlined in circle 2 in (a) and (b)] is mostly removed in (c) and (d). The gray color in circle 2 in (b) is due to band saturation (no data). Gray color for cell concentrations represents 0 counts $\mathrm{L}^{-1}$, and gray color on the MODIS image represents clouds, stray light, or saturation of the MODIS fluorescence bands (especially the 678-nm band, see Ref. 35).

bloom detection, it does impact image appearance, especially to users without too much remote sensing experience. After all, the audience of the FWC bulletins includes users from all backgrounds.

The case studies shown here include all possible cases on the WFS that can cause sediment resuspension, therefore, proving unambiguously that $\mathrm{ABI}$ is a better way than nFLH in bloom detection and false-positive reduction. Indeed, inspection of other similar cases showed the same results. Therefore, in the IRIS ${ }^{15}$ and also in the FWC bulletins, ABI is used in the replacement of $\mathrm{nFLH}$, although for the reason of continuity the name is still kept as nFLH. Likewise, the name of $\mathrm{nFLH}$ is still in the FWC red tide information bulletin distributed to registered users through email subscription.

\section{Discussion}

Through pixel-wise comparison and image inspection for case studies, the modulated MODIS nFLH (i.e., ABI) clearly showed its advantage over the original nFLH. While the purpose of this work was not to compare nFLH with other published algorithms as such as a comparison that has already shown similar statistical performance among those algorithms, ${ }^{9,10}$ the results here also 


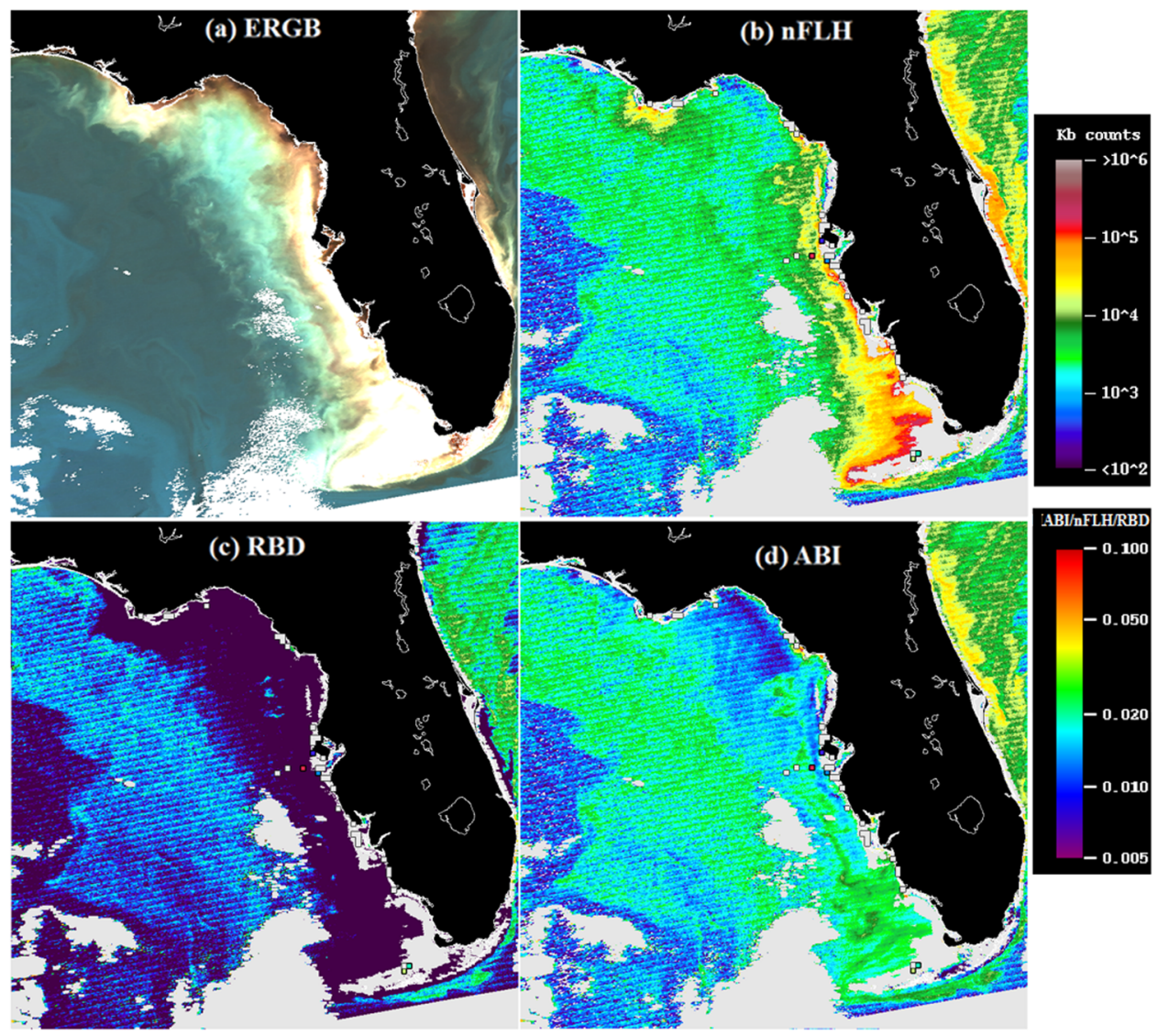

Fig. 5 The performance of three bloom indices for bloom detection during a windstorm. The MODIS images were acquired on day 361 of 2005 , during which wind speed data collected from the NDBC Long Key station $\left(24.844^{\circ} \mathrm{N} 80.864^{\circ} \mathrm{W}\right.$ ) showed maximum wind speed of $8.7 \mathrm{~m} \mathrm{~s}^{-1}$. (a) ERGB; (b) nFLH; (c) RBD; (d) ABI. Annotated in (b)-(d) are K. brevis cell concentrations from water samples collected within one week ( \pm 7 days) of the MODIS image, with the concentration color coded (top color legend). The bottom color legend applies to (b)-(d). The falsepositive detection in sediment-rich waters along the entire WFS [(a) and (b)] has been reduced in both (c) and (d), with more spatially coherent image patterns shown in (d). Gray color for cell concentrations represents 0 counts $\mathrm{L}^{-1}$, and gray color on the MODIS image represents clouds, stray light, or saturation of the MODIS fluorescence bands. ${ }^{35}$

showed slight improvement in ABI as compared with other indices that also use the same 667and 678-nm bands for bloom detection. Overall, pixel-wise comparison showed its slightly improved correlation with $K$. brevis cell counts, and image comparison showed its ability to reduce false-positive detection in sediment-rich waters while maintaining the ability of nFLH for bloom detection in sediment-poor waters. We attribute these improvements to the design of Eq. (4) in further normalizing the nFLH data product against a sediment index, $\operatorname{Rrs}(547)$.

However, similar to $\mathrm{nFLH}, \mathrm{ABI}$ is only an index to detect phytoplankton blooms but it does not differentiate bloom type between $K$. brevis blooms and non-K. brevis blooms (on the WFS, most of the latter are diatom blooms although occasionally there are cyanobacterial blooms). In this regard, $\mathrm{ABI}$ is not as effective as a combination of RBD and $\mathrm{KBBI},{ }^{5}$ or a combination of $\mathrm{Chl}$ anomaly and spectral curvature ${ }^{10}$ in separating $K$. brevis blooms from non-K. brevis blooms. However, as stated earlier, the purpose of using ABI is to provide a simple imagery type for pattern visualization, where integration of field-measured $K$. brevis cell concentrations can confirm whether the elevated ABI values are due to $K$. brevis. Such a purpose has been fulfilled through the IRIS ${ }^{11}$ since the implementation of ABI in 2014, which has been used by the FWC routinely for image interpretation and information distribution. Indeed, although the requirement of concurrent field sampling data may limit its use for many other areas due to lack of a routine 


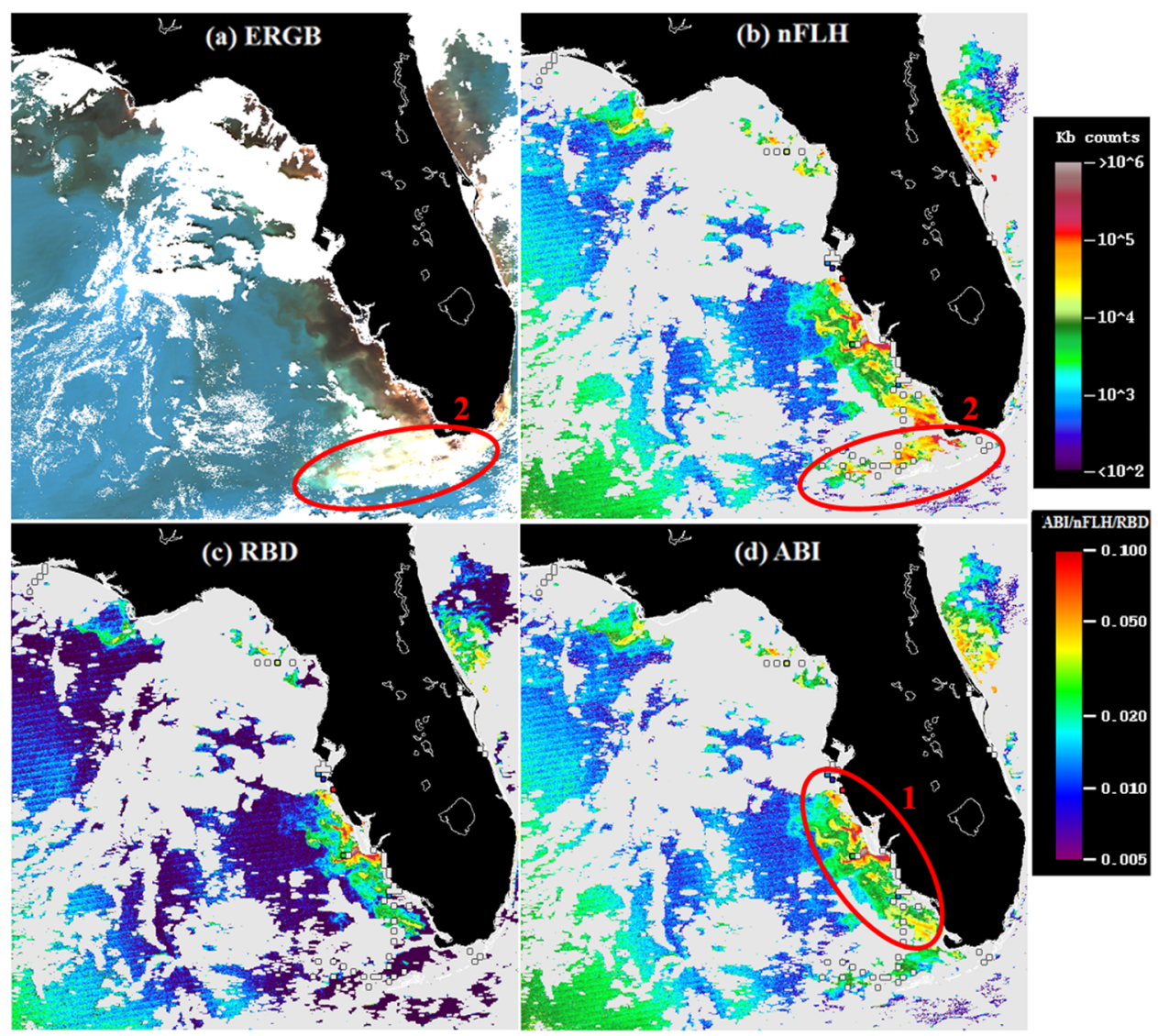

Fig. 6 The performance of three bloom indices for detecting a $K$. brevis bloom and a diatom bloom (outlined in circle 1). The MODIS images were acquired on day 279 of 2003. (a) ERGB; (b) nFLH; (c) RBD; (d) ABI. Annotated in (b)-(d) are K. brevis cell concentrations from water samples collected within 1 week ( \pm 7 days) of the MODIS image, with the concentration color coded (top color legend). The bottom color legend applies to (b)-(d). The false-positive detection in sediment-rich waters north of Florida Keys [circle 2 in (a) and (b)] has been reduced in both (c) and (d), with more spatially coherent image patterns shown in (d). The relatively high values in the lower left corner of (b)-(d) are due to contamination of sun glint because the MODIS 678-nm band is extremely sensitive to sun glint (glint reflectance lower than the threshold to mask image can still contaminate the $\mathrm{nFLH}$ product). Gray color for cell concentrations represents 0 counts $\mathrm{L}^{-1}$, and gray color on the MODIS image represents clouds, stray light, or saturation of the MODIS fluorescence bands.

sampling program for HABs monitoring, at least ABI can serve as an effective bloom index for both sediment-rich and sediment-poor waters. The empirical coefficient $\alpha$ may need to be adjusted through local tuning to account for different sediment types. In case of lack of field data as used in Fig. 2, trial-and-error adjustment may be used until the final ABI image shows similar values in sediment-rich waters as compared with the same waters in adjacent days, assuming benthic algae is negligible. Note that because of the empirical nature, the final value of $\alpha$ ( 80 in our case) is often a compromise between overcorrection and undercorrection. For example, in extremely turbid waters, ABI may not remove the false positives completely (e.g., circle 3 in Fig. 3), but further increases in $\alpha$ would lead to overcorrection [e.g., the elongated blue band around Tampa Bay in Fig. 5(d)].

All analyses in this study were conducted using MODIS/Aqua data only as MODIS/Terra data were considered of having lower quality due to calibration and noise issues. However, an image analyst can usually tell noise from real features, so in routine operations MODIS/Terra images have also been used in order to have a better chance to observe cloud-free and glint-free ocean features. On the other hand, at the time of this writing, both MODIS sensors are aging, with MODIS/Terra being 16-years old and MODIS/Aqua 14-years old. The question is then what to use if they stop functioning. VIIRS (2012 to present) is supposed to continue the 
MODIS observations, yet VIIRS lacks a fluorescence band and thus a similar nFLH or ABI product. However, the VIIRS 672-nm band covers a bandpass of 662 to $682 \mathrm{~nm}$, and therefore, captures a small portion of the fluorescence signal around $685 \mathrm{~nm}$ (25-nm full-width-half-maximum). Qi et al. ${ }^{36}$ showed that such a feature could be used to design a bloom index for dark waters through a 672/551 band ratio of VIIRS. Before the launch of the next-generation ocean color sensors with either hyperspectral capacity (e.g., PACE) or specific fluorescence bands (e.g., Sentinel-3), the use of such a red/green band ratio may be an alternative way to assure relative continuity from MODIS observations in case both MODIS sensors stop functioning.

In any case, neither $\mathrm{nFLH}$ nor $\mathrm{ABI}$ can provide a full solution to bloom detection in optically complex waters, as the ultimate way is to separate nonphytoplankton water constituents from phytoplankton through the use of radiative transfer ${ }^{16,18}$ or semianalytical inversion. ${ }^{37,38}$ However, due to a variety of reasons including large uncertainties in atmospheric correction over turbid coastal waters, these sophisticated methods often do not work well. Adding to this difficulty is the land-adjacent straylight problem in the satellite-retrieved Rrs; such a problem impacts not just the $\mathrm{nFLH}$ or ABI imagery but also all other imagery, regardless of the algorithm used. The consequence is that within 2-km of shoreline there is often no data even when the images have no

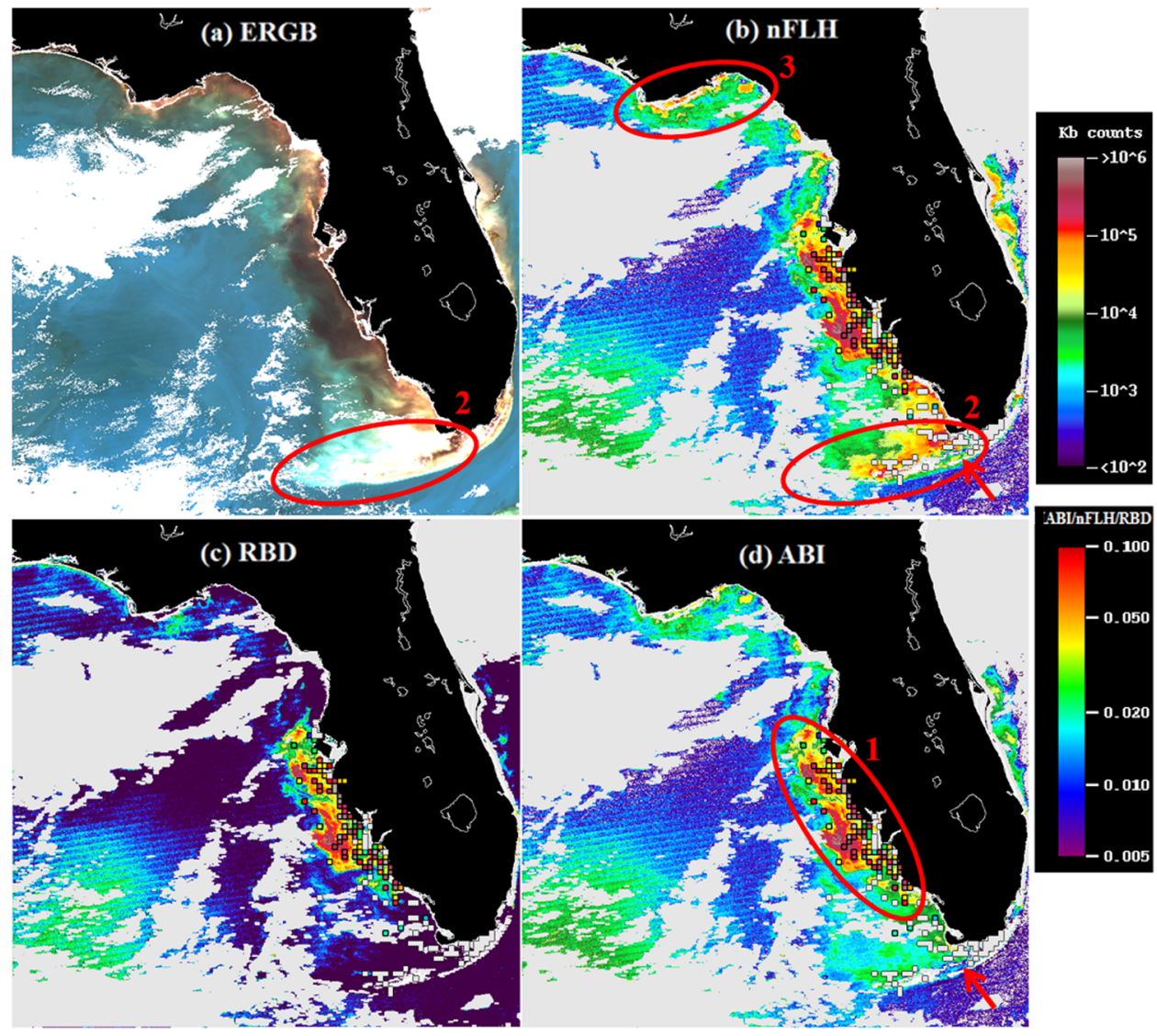

Fig. 7 The performance of three bloom indices in detecting a K. brevis bloom (outlined in circle 1). The MODIS images were acquired on day 280 of 2006. (a) ERGB; (b) nFLH; (c) RBD; (d) ABI. Annotated in (b)-(d) are $K$. brevis cell concentrations from water samples collected within 1 week ( \pm 7 days) of the MODIS image, with the concentration color coded (top color legend). The bottom color legend applies to (b)-(d). The false-positive detection in sediment-rich waters north of Florida Keys [circle 2 in (a) and (b)] has been reduced in both (c) and (d), with more spatially coherent image patterns shown in (d) than in (c). The arrows in (b) and (d) point to shallow-water $(<5 \mathrm{~m})$ locations in nearshore Florida Keys where the falsely elevated $\mathrm{nFLH}$ signals are reduced in $\mathrm{ABI}$. The high nFLH values in the Florida Panhandle (circle 3 ) have also been reduced in (c) and (d), but it is unknown whether these high nFLH values corresponded to high $K$. brevis concentrations. Gray color for cell concentrations represents 0 counts $\mathrm{L}^{-1}$, and gray color on the MODIS image represents clouds, stray light, or saturation of the MODIS fluorescence bands. 
clouds or sun glint. This is clearly shown in nearly all image examples in Figs. 3-7. Future efforts need to focus on minimizing the impact of land-adjacent straylight on data products to maximize the value of satellite data in bloom monitoring.

The use of ABI provides a simple but effective way to further improve nFLH imagery to make them less sensitive to perturbations by resuspended sediments. Although the improvement is not perfect (e.g., there may be still residual errors for extremely turbid waters such as those shown in Fig. 4), its impact on sediment-rich waters can be clearly visualized. Compared to nFLH, ABI is also less sensitive to perturbations by the very shallow bottom $(<5 \mathrm{~m})$ in very clear waters such as those in the Florida Keys [e.g., Fig. 3(b) versus Fig. 3(d); Fig. 7(b) versus Fig. 7(d)]. In such cases of optically shallow waters, although nFLH does not suffer significantly from the bottom interference because of high water attenuation in all three nFLH bands (667, 678 , and $754 \mathrm{~nm}$ ) for water depths $>5 \mathrm{~m},{ }^{39}$ for water depths $<5 \mathrm{~m} \mathrm{nFLH}$ may still be "contaminated" by the shallow bottom when the water is clear. The effect is elevated nFLH values over shallow, clear waters of $<5 \mathrm{~m}$. Such an effect is compensated by basically "normalizing" the nFLH using Rrs(547) through Eq. (4) because Rrs(547) is significantly enhanced by the bottom reflection. Therefore, although Eq. (4) was designed to minimize the impact of sediment resuspension on $\mathrm{nFLH}$, for the same reason, it also reduces the impact of shallow bottom in clear waters. Although not shown explicitly here, the Rrs spectra in the shallow waters $(<5 \mathrm{~m})$ of the Florida Keys showed the bottom interference in nearly all bands, but its impact was reduced in ABI through normalization to Rrs(547). These effects are annotated in Figs. 3(b) and 3(d) and Figs. 7(b) and 7(d).

\section{Conclusion}

The MODIS nFLH data product has been shown effective in detecting blooms in CDOM-rich waters and therefore has been used by the FWC for routine assessment of $K$. brevis HABs in the eastern GOM. However, both radiative transfer and image inspection showed that it was sensitive to perturbations by resuspended sediments, causing false-positive detection. This short article demonstrates a simple but effective method to normalize nFLH data to Rrs(547), resulting in an $\mathrm{ABI}$ that is essentially the same as nFLH for sediment-poor waters but much less sensitive than $\mathrm{nFLH}$ to sediment perturbations. The ABI is also shown to be less sensitive than $\mathrm{nFLH}$ to perturbations by shallow bottom in very clear waters. ABI has been used by the FWC to replace $\mathrm{nFLH}$ in near real-time for routine red tide monitoring, although for continuity reason the name has not been changed. Because of its simple design and the availability of both nFLH and Rrs (547) as the NASA standard data products, the approach may be implemented for other regions for bloom monitoring after local tuning of the empirical correction where nFLH has been found to suffer from the same problems. However, this does not imply that ABI should be used globally over other suggested bloom detection methods (e.g., RBD, KBBI, Chl anomaly, and so on), especially when these methods may have already been used in local systems.

\section{Acknowledgments}

This work was supported by NASA's Ocean Biology and Biogeochemistry Program and Gulf of Mexico Program. MODIS data were provided by the NASA Ocean Biology Processing Group (OBPG) through an online data portal (http://oceancolor.gsfc.nasa.gov). Water samples have been collected by the Florida Fish and Wildlife Commission's Law Enforcement Division and Fish and Wildlife Health Group, which were analyzed by Karen Henschen, Laura Markley, and Katherine Hubbard. We also thank and Karen Atwood of the FWC for preparing and distributing the HAB bulletins. Two anonymous reviewers provided extensive comments and suggestions to help improve the presentation of this work, whose effort is appreciated.

\section{References}

1. J. Walsh et al., "Red tides in the Gulf of Mexico: where, when, and why?" J. Geophys. Res. 111(C11003), 1-46 (2006). 
2. K. A. Steidinger, "Historical perspective on Karenia brevis red tide research in the Gulf of Mexico," Harmful Algae 8(4), 549-561 (2009).

3. US Food and Drug Administration, "National Shellfish Sanitation Program. Guide for the control of molluscan shellfish," Center for Food Safety and Applied Nutrition, US Food and Drug Administration, Washington, DC, pp. 22-28 (2003).

4. K. A. Steidinger et al., "Bloom dynamics and physiology of Gymnodinium breve with emphasis on the Gulf of Mexico," Nato ASI Series Geol. Sci. 41, 133-154 (1998).

5. R. Amin et al., "Novel optical techniques for detecting and classifying toxic dinoflagellate Karenia brevis blooms using satellite imagery," Opt. Express 17(11), 9126-9144 (2009).

6. J. P. Cannizzaro et al., "A novel technique for detection of the toxic dinoflagellate, Karenia brevis, in the Gulf of Mexico from remotely sensed ocean color data," Cont. Shelf Res. 28(1), 137-158 (2008).

7. G. A. Carvalho et al., "Long-term evaluation of three satellite ocean color algorithms for identifying harmful algal blooms (Karenia brevis) along the west coast of Florida: a matchup assessment," Remote Sens. Environ. 115(1), 1-18 (2011).

8. C. Hu et al., "Red tide detection and tracing using MODIS fluorescence data: a regional example in SW Florida coastal waters," Remote Sens. Environ. 97(3), 311-321 (2005).

9. R. Stumpf et al., "Monitoring Karenia brevis blooms in the Gulf of Mexico using satellite ocean color imagery and other data," Harmful Algae 2(2), 147-160 (2003).

10. M. Tomlinson, T. Wynne, and R. Stumpf, "An evaluation of remote sensing techniques for enhanced detection of the toxic dinoflagellate, Karenia brevis," Remote Sens. Environ. 113(3), 598-609 (2009).

11. I. M. Soto, "Harmful Algal Blooms of the West Florida Shelf and Campeche Bank: Visualization and Quantification Using Remote Sensing Methods, University of South Florida, Tampa, Florida (2013).

12. I. M. Soto et al., "Evaluation and optimization of remote sensing techniques for detection of Karenia brevis blooms on the West Florida Shelf," Remote Sens. Environ. 170, 239-254 (2015).

13. National Oceanic and Atmospheric Administration (NOAA), National Ocean Service and the National Environmental Satellite Data and Information Service, "NOAA Harmful Algal Bloom Operational Forecast System (HAB-OFS) bulletins," http://tidesandcurrents.noaa. gov/hab/bulletins.html (11 December 2016).

14. Florida Fish and Wildlife Research Institute, "Florida red tide and other harmful algal blooms (HABs)," https://www.facebook.com/FLHABs (11 December 2016).

15. C. Hu et al., "Developing a smart semantic web with linked data and models for near-real-time monitoring of red tides in the Eastern Gulf of Mexico," IEEE Syst. J. 11(3), 1282-1290 (2016).

16. A. Gilerson et al., "Fluorescence component in the reflectance spectra from coastal waters. Dependence on water composition," Opt. Express 15(24), 15702-15721 (2007).

17. C. Hu et al., "A harmful algal bloom of Karenia brevis in the Northeastern Gulf of Mexico as revealed by MODIS and VIIRS: a comparison," Sensors 15(2), 2873-2887 (2015).

18. D. McKee et al., "Potential impacts of nonalgal materials on water-leaving Sun induced chlorophyll fluorescence signals in coastal waters," Appl. Opt. 46(31), 7720-7729 (2007).

19. C. Hu et al., "Red tide detection in the eastern Gulf of Mexico using MODIS imagery," in Handbook of Satellite Remote Sensing Image Interpretation: Applications for Marine Living Resources Conservation and Management, J. Morales et al., Eds., pp. 95-110, EU PRESPO and IOCCG, Dartmouth, Canada (2011).

20. National Aeronautics and Space Administration Ocean Biology Processing Group (OBPG), http://oceancolor.gsfc.nasa.gov (11 December 2016).

21. Optical Oceanography Laboratory of University of South Florida, "Near real-time integrated red tide information system (IRIS)," http://optics.marine.usf.edu/projects/iris.html (11 December 2016).

22. C. Hu, "A novel ocean color index to detect floating algae in the global oceans," Remote Sens. Environ. 113(10), 2118-2129 (2009).

23. T. T. Wynne et al., "Detecting Karenia brevis blooms and algal resuspension in the western Gulf of Mexico with satellite ocean color imagery," Harmful Algae 4(6), 9921003 (2005). 
24. L. Qi et al., "Influence of particle composition on remote sensing reflectance and MERIS maximum chlorophyll index algorithm: examples From Taihu Lake and Chaohu Lake," IEEE Geosci. Remote Sens. Lett. 12(6), 1170-1174 (2015).

25. B. B. Barnes et al., "Sediment plumes induced by the Port of Miami dredging: analysis and interpretation using Landsat and MODIS data," Remote Sens. Environ. 170, 328-339 (2015).

26. Z. Chen, C. Hu, and F. Muller-Karger, "Monitoring turbidity in Tampa Bay using MODIS/ Aqua 250-m imagery," Remote Sens. Environ. 109(2), 207-220 (2007).

27. L. Feng et al., "Human induced turbidity changes in Poyang Lake between 2000 and 2010: observations from MODIS," J. Geophys. Res. 117, C07006 (2012).

28. R. L. Miller and B. A. McKee, "Using MODIS Terra $250 \mathrm{~m}$ imagery to map concentrations of total suspended matter in coastal waters," Remote Sens. Environ. 93(1), 259-266 (2004).

29. C. Hu, L. Feng, and Z. Lee, "Uncertainties of SeaWiFS and MODIS remote sensing reflectance: implications from clear water measurements," Remote Sens. Environ. 133, 168-182 (2013).

30. A. Sournia, Phytoplankton Manual. Musée National d'Historie Naturelle, United Nations Educational Scientific and Cultural Organization (UNESCO), París (1978).

31. K. A. Steidinger, "Collection, enumeration and identification of free-living marine dinoflagellates," in Toxic Dinoflagellate Blooms, D. L. Taylor and H. H. Seliger, Eds., pp. 435-442, Elsevier/North-Holland, New York (1979).

32. H. Utermöhl, "Zur vervollkommnung der quantitativen phytoplankton-methodik," Mitt. Int. Ver. Theory Angew. Limnol. 9, 1-38 (1958).

33. C. Hu et al., "Linkages between coastal runoff and the Florida keys ecosystem: a study of a dark plume event," Geophys. Res. Lett 31, L15307 (2004).

34. C. Hu, F. E. Muller-Karger, and P. W. Swarzenski, "Hurricanes, submarine groundwater discharge, and Florida's red tides," Geophys. Res. Lett. 33(11), L11601 (2006).

35. C. Hu et al., "Dynamic range and sensitivity requirements of satellite ocean color sensors: learning from the past," Appl. Opt. 51(25), 6045-6062 (2012).

36. L. Qi et al., "VIIRS observations of a Karenia brevis bloom in the Northeastern Gulf of Mexico in the absence of a fluorescence band," IEEE Geosci. Remote Sens. Lett. 12(11), 2213-2217 (2015).

37. Z. Lee, K. L. Carder, and R. A. Arnone, "Deriving inherent optical properties from water color: a multiband quasi-analytical algorithm for optically deep waters," Appl. Opt. 41(27), 5755-5772 (2002).

38. S. Maritorena, D. A. Siegel, and A. R. Peterson, "Optimization of a semianalytical ocean color model for global-scale applications," Appl. Opt. 41(15), 2705-2714 (2002).

39. B. B. Barnes et al., "MODIS-derived spatiotemporal water clarity patterns in optically shallow Florida Keys waters: a new approach to remove bottom contamination," Remote Sens. Environ. 134, 377-391 (2013).

Chuanmin Hu received his $\mathrm{PhD}$ in physics from the University of Miami, Coral Gables, Florida, in 1997. He is currently a professor at the College of Marine Science, University of South Florida, St. Petersburg, Florida, USA. He has been a principal or a co-principal investigator of several projects funded by the U.S. federal and state agencies to study river plumes, harmful and nonharmful algal blooms, oil spills, coastal/inland water quality, and benthic habitat health.

Lian Feng received his $\mathrm{PhD}$ in cartography and geography information systems from Wuhan University, Wuhan, China, in 2013. He is currently a postdoctoral associate at the College of Marine Science, University of South Florida, St. Petersburg, Florida, USA. His research interests include remote sensing of inland and coastal water environments and how these environments are influenced by climate variability and human activities. 